\title{
Three-Dimensional Orientation Microscopy in a Focused Ion Beam-Scanning Electron Microscope: A New Dimension of Microstructure Characterization
}

\author{
S. ZAEFFERER, S.I. WRIGHT, and D. RAABE
}

In the present work, we report our recent progress in the development, optimization, and application of a technique for the three-dimensional (3-D) high-resolution characterization of crystalline microstructures. The technique is based on automated serial sectioning using a focused ion beam (FIB) and characterization of the sections by orientation microscopy based on electron backscatter diffraction (EBSD) in a combined FIB-scanning electron microscope (SEM). On our system, consisting of a Zeiss-Crossbeam FIB-SEM and an EDAX-TSL EBSD system, the technique currently reaches a spatial resolution of $100 \times 100 \times 100 \mathrm{~nm}^{3}$ as a standard, but a resolution of $50 \times 50 \times 50 \mathrm{~nm}^{3}$ seems to be a realistic optimum. The maximum observable volume is on the order of $50 \times 50 \times 50 \mu^{3}$. The technique extends all the powerful features of two-dimensional (2-D) EBSD-based orientation microscopy into the third dimension of space. This allows new parameters of the microstructure to be obtained-for example, the full crystallographic characterization of all kinds of interfaces, including the morphology and the crystallographic indices of the interface planes. The technique is illustrated by four examples, including the characterization of pearlite colonies in a carbon steel, of twins in pseudonanocrystalline NiCo thin films, the description of deformation patterns formed under nanoindents in copper single crystals, and the characterization of fatigue cracks in an aluminum alloy. In view of these examples, we discuss the possibilities and limits of the technique. Furthermore, we give an extensive overview of parallel developments of 3-D orientation microscopy (with a focus on the EBSD-based techniques) in other groups.

DOI: $10.1007 / \mathrm{s} 11661-007-9418-9$

(C) The Minerals, Metals \& Materials Society and ASM International 2008

\section{INTRODUCTION}

\section{A. Motivation: Why Perform Three-Dimensional Investigation?}

MetallogRAPHIC techniques for the characterization of microstructures of materials are usually applied to two-dimensional (2-D) planes cut through a sample. Statistical stereological techniques can be used to gain insight into the three-dimensional (3-D) aspects of microstructure.$^{[1,2]}$ However, there are also a large number of cases in which a true 3-D characterization of a sample volume is critical to a correct understanding of microstructure formation mechanisms or particular material properties. Cases in which 3-D information is of great importance include, for example, the true size and shape of grains for grain growth investigations, the size and shape of strain fields for studies on deformation, and the distribution and position of nuclei for recrystallization investigations. An example of informa-

S. ZAEFFERER, Head of the Microscopy and Diffraction Group, and D. RAABE, Department Director, are with the Max Planck Institute for Iron Research (MPIE), D-40235, Düsseldorf, Germany. Contact e-mail: s.zaefferer@mpie.de S.I. WRIGHT, Director of Applications Science and Software, is with EDAX-TSL, Draper, UT 84020

Manuscript submitted May 22, 2007.

Article published online January 4, 2008 tion that is completely missing in 2-D microstructure observations is the full crystallographic characterization of all kinds of interfaces. In 2-D orientation microscopy, the misorientation across a boundary can be determined; however, since the spatial orientation of the boundary plane is unknown, the orientation of the boundary plane relative to the crystal lattices separated by the plane cannot be determined. This information is of great importance for the investigation of phase transformations, grain growth processes, and intergranular fracture.

Principally, the 3-D characterization of microstructures can be performed by two different approaches, either by serial sectioning or by observation with some sort of transmissive radiation. The latter techniques obtain the 3-D information from bulk samples, either by reconstruction from a large number of extinction images taken from different directions of the sample or by a raytracing technique. The ray-tracing techniques allow crystallographic information to be obtained. They have undergone intensive development in recent times in various groups, as in, for example, References 3 through 6 , and made impressive progress. The current spatial resolution is on the order of the volumes of some cubic microns and the method has been shown to yield in-situ observations of recrystallization and deformation processes of cubic materials, as in, for example, References 7 and 8. 


\section{B. Tomography by Serial Sectioning}

Significantly simpler than the bulk methods, yet comparably powerful (and partly complementary) methods for the 3-D characterization of crystalline materials are the serial sectioning techniques, which are tomographic techniques, in the word's original meaning. The technique is simply composed of cutting material slices with some cutting technique, recording the structure of these slices with an appropriate microscopic or macroscopic recording technique, and reconstructing the 3-D structure, e.g., by stacking the recorded images. Serial sectioning is applicable to a very wide range of materials and material problems; the only serious disadvantage is that the method is destructive.

For serial sectioning, numerous and different methods can be imagined, e.g., mechanical cutting, grinding, or polishing; chemical polishing; or laser or electrical discharge ablation. For observation of the serial sections, all kinds of microscopic techniques are available, e.g., light optical observations following some etching procedure or scanning electron microscope (SEM) observations using contrast created by backscattered electrons (BSEs). The main challenges associated with many of the sectioning methods are controlling the sectioning depth, obtaining flat and parallel surfaces, and correctly redetecting and aligning the area of observation. On the observation side, the main obstacle is that most observation techniques do not allow certain microstructural features, such as grain boundaries or individual grains, to be separated, because the contrast conditions are not well enough defined. Finally, most serial sectioning methods are extremely laborious. Examples of various serial sectioning methods have been compiled in one of our previous publications. ${ }^{[9]}$

A technique that avoids, in an ideal way, all of these problems with sectioning and imaging is the combination of serial sectioning with an ion beam- and electron backscatter diffraction (EBSD)-based orientation microscopy in a combined focused ion beam (FIB)SEM. The FIB consists of $\mathrm{Ga}^{+}$ions, accelerated to, for example, $30 \mathrm{keV}$. The impact of the beam onto a sample leads to localized sputtering of the target material and can therefore be used to perform cuts into the material of few nanometers in width. Irradiating a material surface in grazing incidence allows the preparation of smooth surfaces that show little radiation damage. ${ }^{[10-12]}$ This technique has been used extensively for the preparation of transmission electron microscopy (TEM) samples, as well as for the preparation of flat surfaces for metallographic investigations. Through the sputtering of subsequent parallel surfaces, serial sections can be created that may be separated by precisely defined distances of some $10 \mathrm{~nm}$ up to several microns. A variety of articles describe such a technique for the preparation of serial sections or TEM samples, e.g., References 12 through 15.

For observation of the microstructures of the serial sections, orientation microscopy using the EBSD technique in the SEM is an excellent method. ${ }^{[16-18]}$ Here, different phases and grains can be clearly recognized and quantitatively described. Grain boundaries can be marked and easily classified according to their crystallographic character. Each feature can be separated and displayed individually. Areas (or volumes in 3-D) belonging to the same grain in different sections can be easily assembled by their similar orientation. Orientation microscopy is, therefore, the pre-eminent method for 3-D microstructure characterization based on serial sectioning.

The combination of EBSD-based orientation microscopy with serial sectioning is not completely new, but is currently under strong development. The recent viewpoint set in Reference 19 documents examples. In addition, the combination of orientation microscopy with serial sectioning by FIB has been described previously. ${ }^{[19-23]}$

The aim of the present article is to give a comprehensive overview on the emerging technique of 3-D orientation microscopy based on serial sectioning, using a FIB- and 2-D EBSD-based orientation microscopy. We illustrate the technique by means of our own developments, which include, in fact, a new geometrical setup that has advantages over setups developed thus far by other groups. The potential of the technique for materials science is demonstrated by some of our recent experimental results. Other results obtained by a manual procedure have been documented already in earlier publications. ${ }^{[9,24,25]}$

\section{PRINCIPLE OF 3-D CHARACTERIZATION IN A FIB-SEM}

\section{A. The Geometrical Setup of the System}

The system described here has been developed on a Zeiss-Crossbeam (Carl Zeiss SMT AG, Oberkochen, Germany, CrossBeam (R)) XB 1540 FIB-SEM, which consists of a Gemini-type field emission gun (FEG) electron column and an Orsay Physics (Orsay Physics FIB is a part of the CrossBeam instrument) ion beam column mounted 54 deg from the vertical. For EBSD, a Digiview camera of EDAX-TSL (EDAX/TSL, Draper, UT) is mounted on a motorized slide; this allows the camera to be driven under the computer control to its operating position in the chamber and withdrawn as needed. An energy-dispersive X-ray spectroscopy (EDX) detector is mounted such that the EBSD and EDX signals can be simultaneously detected. The geometric arrangement of the system is schematically shown in Figure 1(a). In our system, in contrast to all other systems already available, the EBSD camera and EDX detector are mounted opposite the ion column.

For EBSD, the investigated surface is usually tilted 70 deg from the horizontal position. As illustrated in Figure 1(a), this requires a sample tilt between grazingincidence ion milling and EBSD of $34 \mathrm{deg}$. We therefore call this setup the "tilt" setup, due to the angular limitations of the stage currently used and due to the fact that, in the EBSD position, the EBSD camera is positioned very close to the sample (approximately $20 \mathrm{~mm}$ ) and the sample must be mounted on a relatively high pretilted stage. Therefore, the sampled area cannot 


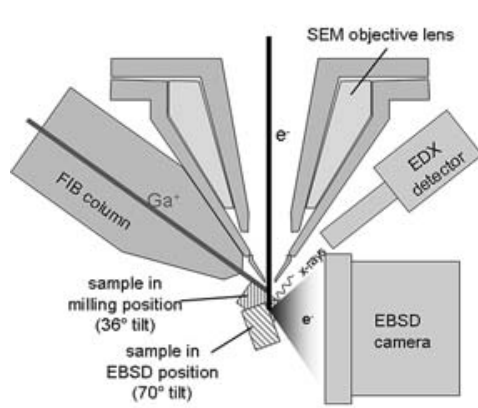

(a)

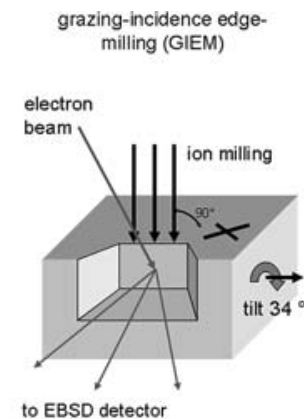

(b)

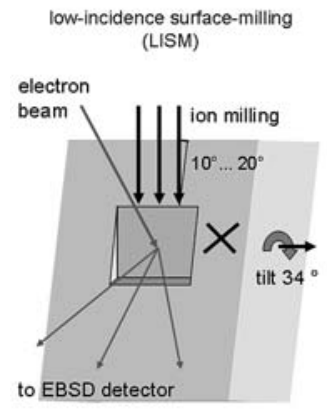

(c)

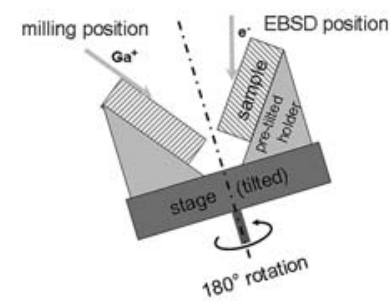

(d)
Fig. 1-Schematics of the geometry of the 3-D procedure. (a) Cross section through the instrument chamber, showing the sample positions for milling and EBSD analysis in the tilt setup. To change between the two positions, the sample need only be tilted and moved in the $y$ direction. During milling, the EBSD camera is retracted to the chamber wall. Working distance for milling is $8 \mathrm{~mm}$; for EBSD, it is $13 \mathrm{~mm}$. The camera is positioned at a distance of $22 \mathrm{~mm}$ from the sample. $(b)$ and $(c)$ Schematics of different variations of the tilt geometry: (b) the GIEM method and (c) the LISM method. For both schemes, the black cross indicates the marker for image alignment that is milled into the sample before the process begins. $(d)$ The rotation geometry. Both the EBSD and milling positions are shown together. A tilt-free setup requires a pretilted holder mounted on a pretilted stage.

be brought into the eucentric tilt position of the stage. Consequently, each sample tilt must be combined with a $y$ movement of the stage.

Using the tilt setup, two measurement strategies can be realized. The first, called the grazing-incidence edgemilling (GIEM) method, is illustrated in Figure 1(b). The sample is prepared by grinding and polishing to produce a sharp rectangular edge. Milling is then performed under grazing incidence to one surface at this sample edge. After milling has created a smooth surface at the desired distance from the sample surface, the sample is moved into EBSD position by a 34-deg tilt and the appropriate $y$ movement of the stage. The EBSD is performed on this surface. After EBSD has completed, the sample is returned to the milling position and the next cut is performed. This method has the advantage of being easy to set up and allowing the investigation of large volumes. In contrast, the microstructure to be investigated must be close to the edge, which is not always easily achieved or possible at all. In this case, a second, alternative method may be applied, the low-incidence surface-milling (LISM) method, which

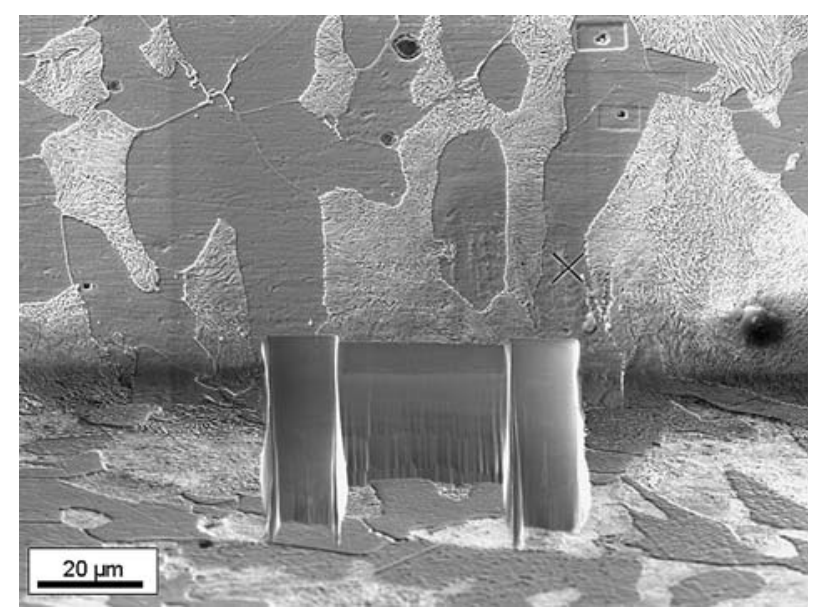

Fig. 2-Microstructure of the investigated pearlitic steel sample after milling (no image has been taken before milling). Image shows the edge of the sample as viewed in EBSD position (investigated area at $20 \mathrm{deg}$ to the primary beam). The cross on the right side of the milled area is the alignment marker. The two deeper milled areas, left and right, have been milled with a coarse beam, to avoid shadowing of the EBSD camera.

is illustrated in Figure 1(c): the initial sample preparation must produce a fine polished surface. For milling on the free surface, the sample is tilted such that the ion beam enters the surface at an angle of 10 to $20 \mathrm{deg}$. As in the GIEM method, the sample is subsequently tilted by $34 \mathrm{deg}$, and EBSD is performed on the milled surface. The advantage of this method is that virtually any volume close to the sample surface can be investigated. The disadvantage is that the milling area needs to be significantly larger than in the case of GIEM, in order to avoid shadowing of the EBSD screen.

In an alternative geometrical setup that has been adopted by other groups (e.g., Reference 21) — we call it the "rotation" setup - the EBSD camera is situated below the FIB gun. The movement from milling to EBSD position has then to be accomplished by a 180 -deg sample rotation and some $x$ and $y$ movements, if the observed position is not precisely on the rotation center. If the sample is mounted on a pretilted holder and the stage is additionally tilted such that the rotation axis is positioned halfway between the EBSD and the milling position, then it is possible to obtain a situation in which no further sample tilt is required to change between the two characteristic positions. This situation, illustrated in Figure 2(c), may allow the achievement of a higher stagepositioning accuracy than does the tilt setup; however, this advantage is not really evident, as we will show by a comparison of the two possible setups in Section IV.

For both setups, the size and shape of the milled area is determined, on the one hand, by the size of the investigated surface. On the other hand, it has to be considered that the edge of the milled area projects a shadow onto the EBSD camera. The shadow increases with increasing distance of the milled area from the free sample surface and decreasing distance between the EBSD camera and the sample surface. For high-speed EBSD, a large acceptance angle of the diffraction pattern is beneficial. At a camera position of $20 \mathrm{~mm}$, 
we obtain an acceptance angle of approximately 120 deg. This means that the milled area must be bound by at least 60-deg angles on all three edges, in order to allow shadow-free diffraction patterns (Figure 13). This additionally milled volume may significantly increase milling time at large milling depths or when applying the LISM method. A reasonable alternative is to remove the shadowing areas with a large ion beam current and mill only the investigated surface with a small and, therefore, finely focused ion current. This is accomplished in our system by setting up a milling list that holds a number of user-defined milling areas.

For both measurement strategies, a position marker is required that allows accurate positioning of the sample after movement from EBSD observation to milling or vice versa. As a position marker, we use a cross that is milled next to the measured area into the sample surface. For the tilt setup, a single marker is sufficient, because only translations are needed (no sample rotation is applied). In contrast, for the rotation setup, two markers are used. The accuracy of sample positioning will be discussed further in Section IV.

\section{B. Automatic 3-D Orientation Microscopy}

Fully running the 3-D milling and EBSD cycle automatically requires a number of instruments and processes to be synchronized: for orientation microscopy, the SEM, the EBSD program, the camera slide controller, the camera controller, and the electron image drift correction program need to cooperate. For sectioning, the FIB and the FIB image drift correction program are required. Finally, for changing between the milling and the EBSD position, the sample stage needs to be accurately controlled. All participating processes are controlled by one 3-D measurement program. Once the software is configured, it runs the milling and EBSD cycle until a predefined number of cycles has been reached. A cycle consists of the following steps.

(1) The EBSD camera is moved into position in the chamber.

(2) The position marker is detected by image cross correlation and the sample brought to its reference position using beam shift functions.

(3) A reference image is taken in the EBSD position.

(4) Orientation mapping is performed using the predefined camera control parameters.

(5) After orientation mapping has been fully accomplished, the camera is retracted, the stage is moved to the predefined milling position, and the FIB gun is switched on if it was off.

(6) The microscope is switched to FIB mode and the position marker is detected by cross correlation. A shift of the FIB beam brings the milling area into reference position.

(7) A reference image is taken, using the FIB beam to document the milling progress from the FIB position.

(8) The FIB switches to milling mode, steps the beam forward to the desired $z$-step size, and performs the milling list.
(9) Once milling is finished, the FIB gun is switched off, if requested, and the stage is moved back to the predefined EBSD position.

Automatic 3-D orientation microscopy puts high demands on the measurement process: (1) the stage positioning has to be accurate enough to allow redetection of the marker in each position in the limits of the beam shift controls, (2) the precision of the stage tilt must be high enough to avoid any visible distortion of the field of view, (3) stage drift after sample repositioning must be minimized and the stage must reliably come to a complete stop in the range of tens of seconds, (4) the position-correction algorithm must be precise enough to achieve the desired resolution, and (5) the SEM and the FIB must work stably over long measurement times. All of these demands are fulfilled on the system presented here. We discuss the limits in more detail in Section IV.

\section{Software for 3-D Analysis}

For the analysis of 3-D data, rudimentary software has been developed thus far. It allows any position of the measured volume in space to be viewed through the sequential display of 2-D microstructure images. For preparation of the individual slice images, the batchprocessing function of the EDAX-TSL OIM Analysis*

*OIM Analysis is a trade mark of EDAX/TSL, Draper, UT.

software package is used. This function allows the application of similar operations to any number of measured data sets - for example, the rotation of the reference system, cleanup, map construction, and saving of images to disk.

Perhaps the most important advantage of 3-D orientation microscopy over 2-D systems is the possibility of determining the crystallography and morphology of interfaces. Our 3-D software, therefore, contains a tool that allows the manual selection of grain boundaries or other interfaces. Subsequently, the local grain-boundary normals in the sample and the crystal reference systems are calculated.

An important precondition for the clear rendering of 3 -D data is the precise alignment of the slices. Although this is achieved to a large extent during the data acquisition process, the data still show misalignment between the slices. We, therefore, developed an alignment tool that is based on the calculation of a correlation function:

$$
C i j=\sum_{i j}\left|(\varphi 1, \Phi, \varphi 2)_{t, x, y}-(\varphi 1, \Phi, \varphi 2)_{w, x+i, y+j}\right|
$$

which means that the values of the three Euler angles at a position $x, y$ in the template orientation map are subtracted one by one from those at the position $x+i$, $y+j$ in the working map. The position at which the sum of all differences, $C i j$, is at a minimum produces the best alignment. Note that, before Cij is calculated, 
the Euler angles must be reduced to a single symmetric variant.

Other groups have developed more advanced software. A well-known software package for 3-D rendering is, for example, the program IMOD (IMOD package is Copyright (c) 1994-2006 by the Boulder Laboratory for 3-Dimensional Electron Microscopy of Cells and the Regents of the University of Colorado), which is based on the construction of 3-D wire-frame models from 2-D cuts. ${ }^{[26]}$ We have used the software ourselves for the presentation of 3-D data; ${ }^{[9]}$ however, a serious disadvantage is the enormous manual work required for model preparation. Others have developed software that allows the selection of 3-D grains and the determination of volume properties of these grains, e.g., Reference 21 . A very powerful 3-D representation of grain boundaries has been presented by Rowenhorst et al., ${ }^{[2]}$ who use a coloring of grain boundaries according to the position of the local crystallographic normal vector in a standard orientation triangle.

\section{APPLICATION EXAMPLES}

In this section, four selected examples of 3-D materials analysis are presented, each measured using the described system in tilt setup. The selection of the samples has been made such that the strengths and the limits of the system can be well demonstrated. The strengths lie clearly in the ease of operation and in the spatial resolution-in the precise definition of microstructure, which allows, for example, deformed or multiphase material to be characterized. The limits are confined by the relatively small investigable volume and by possible material/microstructure alteration due to ion beam irradiation.

For all measurements presented, the ion beam column was operated at an accelerating voltage of $30 \mathrm{kV}$, with beam currents between 100 and $2 \mathrm{nA}$. For EBSD, an electron accelerating voltage of $15 \mathrm{kV}$ was used. The EBSD camera was always positioned approximately $20 \mathrm{~mm}$ away from the sample. All EBSD measurements were performed at a high measurement rate of 65 to 70 patterns per second. This could only be accomplished by minimizing the pattern indexing calculation time. To this end, in multiphase materials, only the most simple phase was indexed online. The complete indexing for all phases was then performed offline from the recorded Hough-peak data, using the full crystallographic information.

\section{A. Pearlite}

Pearlite is a lamellar arrangement of ferrite $(\alpha-\mathrm{Fe}$ with a bcc lattice structure) and cementite $\left(\mathrm{Fe}_{3} \mathrm{C}\right)$; in 2-D investigations, pearlite often exhibits the characteristic bending of the lamella structure that is related to a significant orientation change of the ferrite and likely also of the cementite. It is not clear whether the information about the cementite is accurate, because the crystal orientation of cementite is very difficult to measure by electron diffraction techniques, due to its low structure factors. The occurrence of bending and of the related orientation change is an interesting hint of the physical mechanism of phase transformation. A number of models have been proposed to explain the pearlite formation, e.g., References 28 through 30. A 3-D investigation of the arrangement of pearlite lamellae yields new and otherwise inaccessible information. First, the direction of maximum orientation gradients may be determined and spatially correlated with the strength of lamella curvature. Second, the ferrite-cementite phase boundary might be precisely characterized with respect to the ferrite grain-boundary normal.

For our 3-D investigation, a simple high-carbon steel Fe 0.49 pet $\mathrm{C}$ sample has been selected. The sample was heated to $950{ }^{\circ} \mathrm{C}$ and then furnace cooled at a rate of $0.1{ }^{\circ} \mathrm{C} / \mathrm{s}$. Figure 2 shows the remaining microstructure of the pearlite colonies after milling. The cementite lamella (white) had a width of 100 to $200 \mathrm{~nm}$ and a distance varying between 200 and $400 \mathrm{~nm}$. The measurement system has been set up for a GIEM measurement. Fifty slices of $30 \times 20 \mu \mathrm{m}^{2}$ were milled with a step size of $100 \mathrm{~nm}$. Orientation mapping was performed using a lateral step size of $100 \mathrm{~nm}$ as well. Only the ferrite phase was indexed, because cementite did not produce any indexable patterns, but appeared as simply a degradation of the ferrite patterns. The time for one complete cycle was approximately 35 minutes, including 16 minutes for milling and 15 minutes for EBSD mapping.

Figure 3 displays the result of the measurement in the form of a 3-D orientation map, in which the color of each voxel (volume pixel) is a combination of the diffraction pattern quality, which defines the brightness of the voxel and the orientation of the measurement plane normal this, in turn, defines the color, according to the inverse pole figure (IPF) color triangle. Though cementite could not be indexed, the reduced ferrite diffraction pattern quality indicates the position of the lamellae. The figure shows the high alignment accuracy of the measurement: at many positions, it is possible to

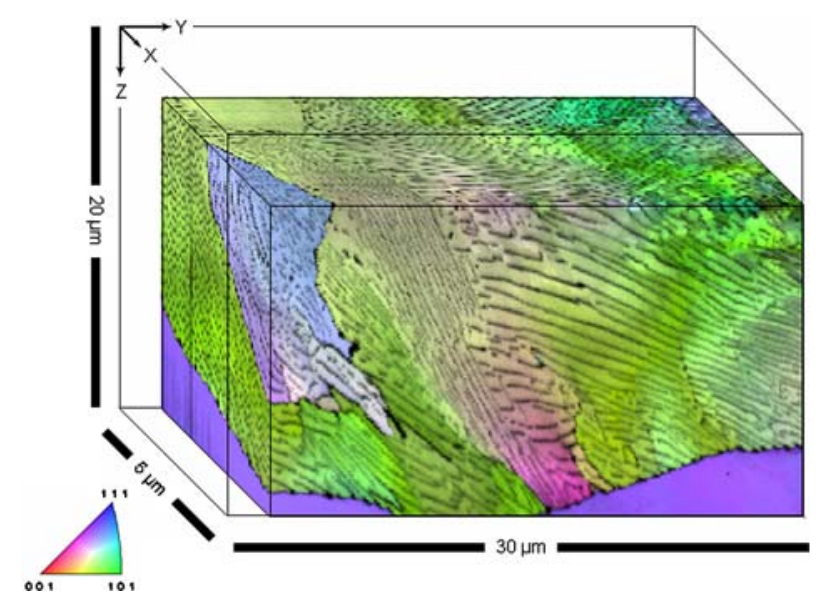

Fig. 3-The 3-D microstructure of a pearlite block. The color is composed of image quality (gray value) and a color code for the crystal direction parallel to the $x$ axis of the sample. The outer block indicates the full measured volume. A part has been cut off because of low pattern quality in these areas. 
track the pearlite lamella through all three dimensions. Nevertheless, in areas in which the lamellae are close together, their exact direction is not clear. We discuss problems of alignment in Section IV. The strong orientation change across the volume is displayed in Figure 4(a), in which the color code denotes the misorientation angle of a given voxel with respect to a reference orientation. As reference, the orientation of the lamella-free area in the upper back corner is selected. The maximum indicated misorientation is $15 \mathrm{deg}$.

Figure 3 shows that the observed pearlite consists of two grains and several pearlite colonies. While strong continuous ferrite orientation gradients run parallel to the pearlite lamellae, generally no continuous orientation change occurs perpendicular to the lamellae; in contrast, small-angle grain boundaries are found to lie mainly parallel to the lamellae. The convergence of the orientation gradient with the lamella position shows that the orientation gradient is directly related to the growth process of the lamellae.
The results of some first interface investigations are demonstrated in Figure 4: two colonies of pearlite lamellae, 1 to 6 and 7 to 10, have been investigated. As indicated by the pole figures in Figure 4(b), in each of the lamellae groups, the ferrite crystals continuously rotate about a (111) plane. The rotation pole, however, is different for both groups. The positions of the lamellae normals relative to the sample reference frame are displayed in the pole figure in Figure 4(c) and, relative to the ferrite crystal orientation, in IPFs in Figure 4(d). The IPFs show that the ferrite phase boundaries are close to (111), albeit with a significant deviation of up to $16 \mathrm{deg}$. It has been shown by TEM investigations that the atomic habit plane of ferrite-

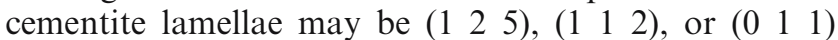
(References 31 and 32, for example), depending on the orientation relationship between both phases. While the (111) plane determined here deviates considerably from these results, it has, in fact, been shown that there is not necessarily any correspondence between the atomic habit plane and the macroscopic one. ${ }^{[33]}$ Furthermore,
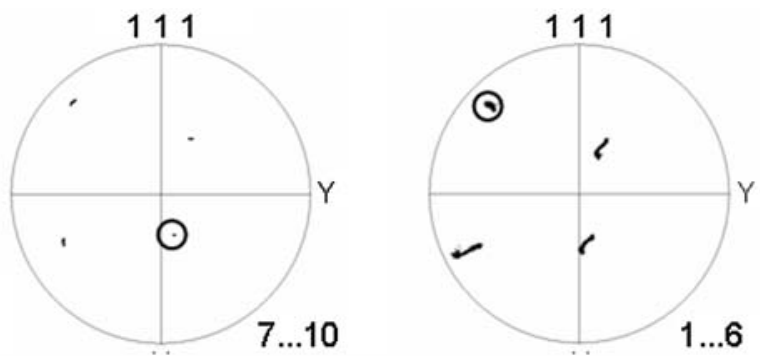

(b)

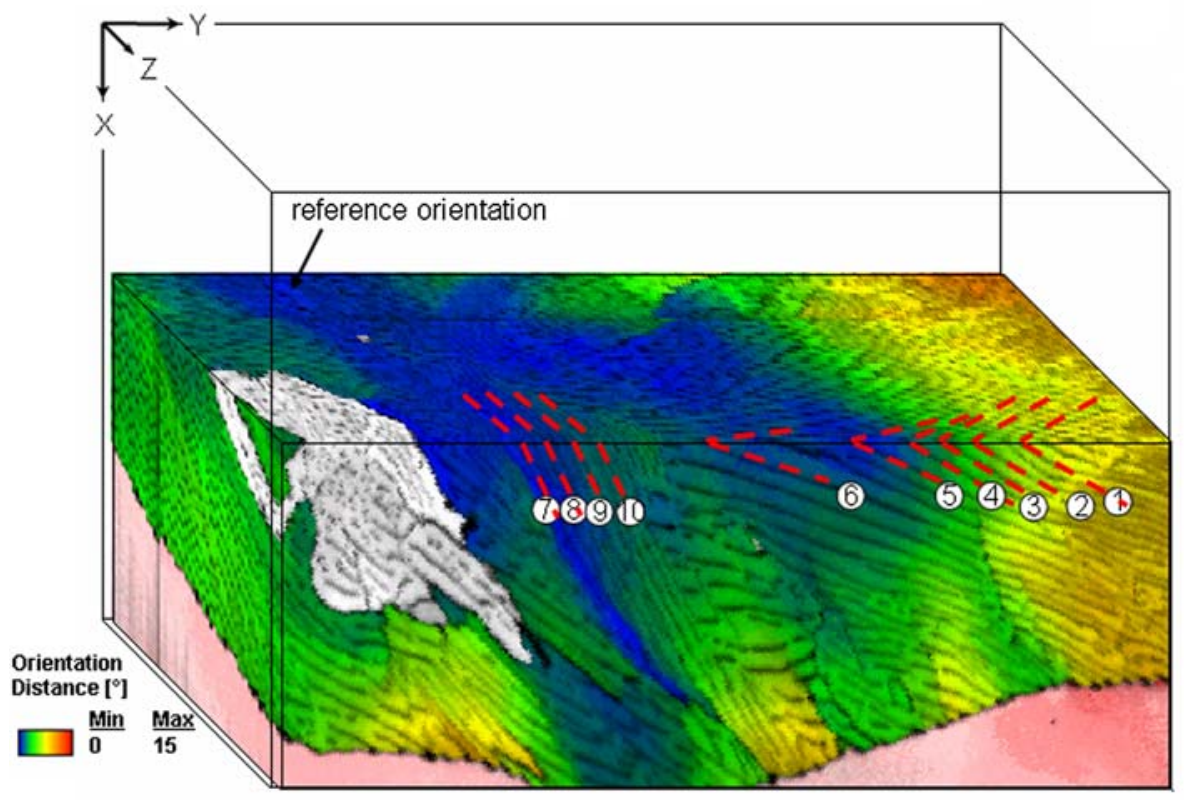

(a)

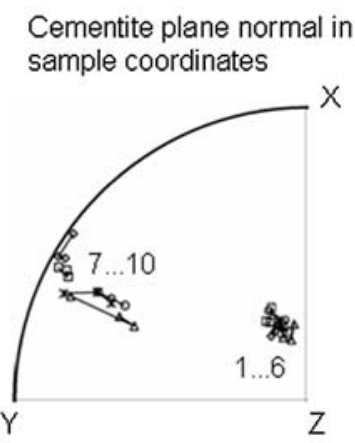

(c)

Cementite plane normal in ferrite crystal coordinates
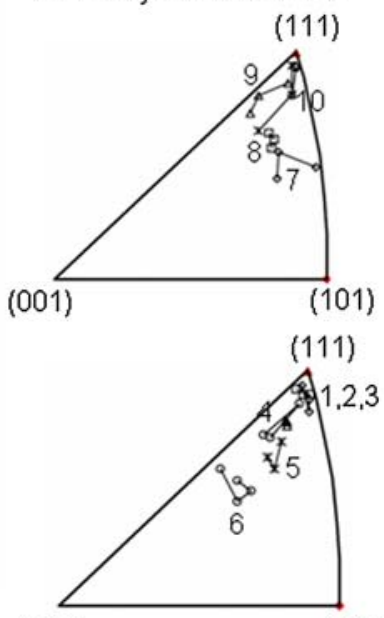

(001)

(d)
(101)

Fig. 4 -Ferrite-cementite interface analysis: $(a)$ microstructure with some cementite lamellae marked; $(b)(111)$ pole figure of the area of lamellae 7 to 10 (left) and 1 to 6 (right), indicating that each pearlite colony is characterized by one common (111) pole as rotation axis, with the common pole marked by a circle; $(c)$ stereographic projection of the cementite lamellae plane normals; and $(d)$ IPF of the crystallographic ferrite plane normals of the cementite lamellae. For both colonies, ferrite plane normals close to (111) are found, although with a significant deviation. For (c) and (d), each lamella position has been measured several times, as indicated by similar symbols connected by lines. 
from the present results, it seems that there is also no clear relationship between the crystal orientation and the macroscopic habit plane.

\section{B. "Nanocrystalline" NiCo Deposits}

Electrodeposition from aqueous solution has been intensively studied as a method to create metallic films with a nanocrystalline microstructure. ${ }^{[34-39]}$ The deposition parameters, such as solution composition, current density, and bath movement, strongly influence the developing microstructure. The exact mechanisms of microstructure formation are still largely unknown, in part because a precise characterization of the microstructure has rarely been undertaken. In a recently published work, ${ }^{[40]}$ we applied EBSD-based high-resolution orientation mapping to obtain a deeper knowledge of the microstructure of electrodeposited thin films of NiCo. Most of the investigated films consist of a large volume fraction of columnar crystals with cross sections in the submicrometer range but lengths on the order of more than $30 \mu \mathrm{m}$. For all columnar grains, the $\langle 11 \overline{2} 0\rangle$ direction is close to the growth direction of the crystal column. Only in niches beside these crystals do areas of truly nanocrystalline material exist. We found, furthermore, that the elongated crystals are, in most cases, growing in triples of three parallel crystals related to each other by a twin orientation relationship. The existence of these triples can be seen on the free grown surface of the electrodeposited material in the form of threefold pyramids. We suspect that the existence of twins stabilizes the growth of columnar grains by the formation of low-energy boundaries.

Analysis using 3-D orientation microscopy was completed on a full $100-\mu \mathrm{m}$ wide cross section of an electrodeposited material. The sample was manually ground and polished on the cross sections to produce a sharp rectangular edge. Figure 5 shows the sample

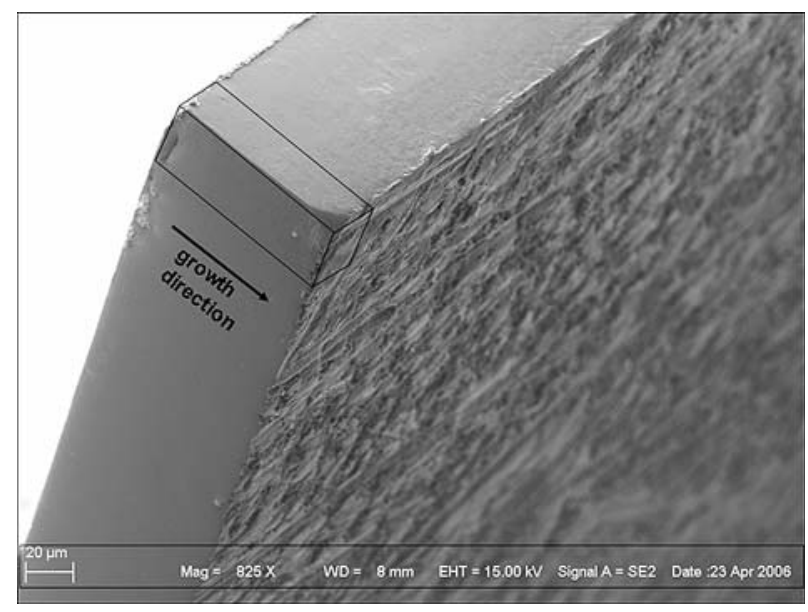

Fig. 5-Secondary electron image of the cross section of the $\mathrm{NiCo}$ thin film ready for 3-D orientation microscopy. The investigated volume is indicated by a black line frame; the growth direction is indicated by an arrow. The rectangular edge has been prepared by mechanical grinding and polishing. Note that, due to grinding, the typical growth surface has been erased. before milling. The 3-D orientation microscopy was performed using the GIEM method. A step size of $100 \mathrm{~nm}$ in all dimensions was selected and 60 maps covering a volume of $83 \times 16 \times 6 \mu^{3}$ were measured within less than two days. The results are shown in Figure 6(a), in the form of a material block colored according to the IPF in a direction perpendicular to the growth direction. The usual large-angle grain boundaries are colored black, while all possible twin boundaries are displayed as white lines. In the center of the block, a part has been cut out to display a typical twin triple. In Figure 6(b), the block is cut along the plane that contains the twin triple indicated in Figure 7(a). The triple is not growing straight, but is visibly quite heavily curved. Together with this curvature, the orientation of the triple changes, as shown in Figure 6(c), which displays the deviation of the macroscopic growth direction from the $\langle 11 \overline{2} 0\rangle$ crystal direction. The crystal orientation is continuously deflected by the regular occurrence of lattice defects of some sort. The nature of these lattice defects, visible in form of inclined stripes in Figure 6(c), is not yet clear. In the center of the measured block, the twin triple consists of three grains with two 56-deg $\langle 11 \overline{2} 0\rangle$ twin relations between them and an approximately 66-deg $\langle 11 \overline{2} 0\rangle$ relationship on the remaining boundary (Figure 7 ). From our earlier 2-D investigations, ${ }^{[40]}$ we proposed the following formation mechanism for the triple: a primary crystal (1) twins independently with the same twin relationship into two twin variants, (2) and (3) such that all three crystals show a $\langle 11 \overline{2} 0\rangle$ direction parallel to the macroscopic growth direction optimum for quick growth. The boundary between the crystals (2) and (3) is then automatically fixed to a 66-deg $\langle 11 \overline{2} 0\rangle$ relationship. In the light of the new 3-D data, this explanation no longer seems totally correct. Following the triple in the growth direction, we first notice that the grains (1) and (2) exist as twins with a sharp 57-deg $\langle 11 \overline{2} 0\rangle$ relation already when they first enter into the measurement area (marked as point A in Figure 6(b)) (note that grain (2) is not visible in the figure, as it is on top of grain (1)). At point B, grain (3) nucleates and grows for the first approximately $10 \mu \mathrm{m}$ as a very thin tube parallel to grains (1) and (2). At the position at which the growth direction), grain (3) also expands and grows larger (point C). At this position, we applied the 3-D analysis program to determine the grain-boundary characteristics. The results are displayed in Figure 7: between grains (1) and (2), we find the $\{10 \overline{1} 1\}$ twin boundary, typical for these twins. ${ }^{[41]}$ Grain (3), although it shows a precise twin orientation relation with grain (2), does not have a twin boundary with the latter, i.e., the plane indices on both sides of the grain boundary are different, the index of grain (2) being very precisely the (0001) basal plane and the other index being some quite highly indexed one. The same is true for the boundary with grain (1), although this grain boundary even does not show any typical twin orientation relationship. We conclude that grain (3) is a twin of grain (2), but its boundary is incoherent and therefore of a quite different quality than that between (1) and (2). We suppose, furthermore, that the (0001) basal 


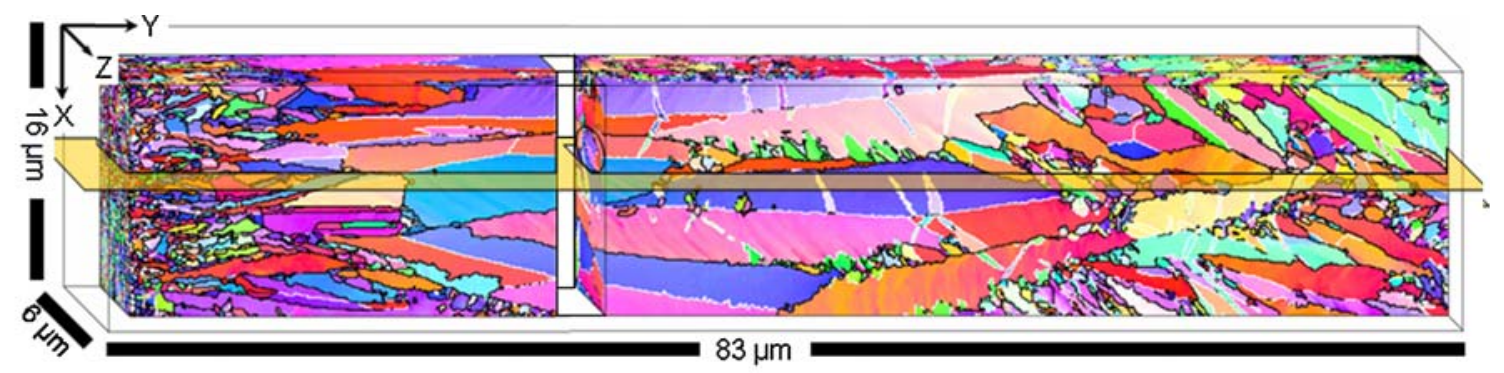

(a)

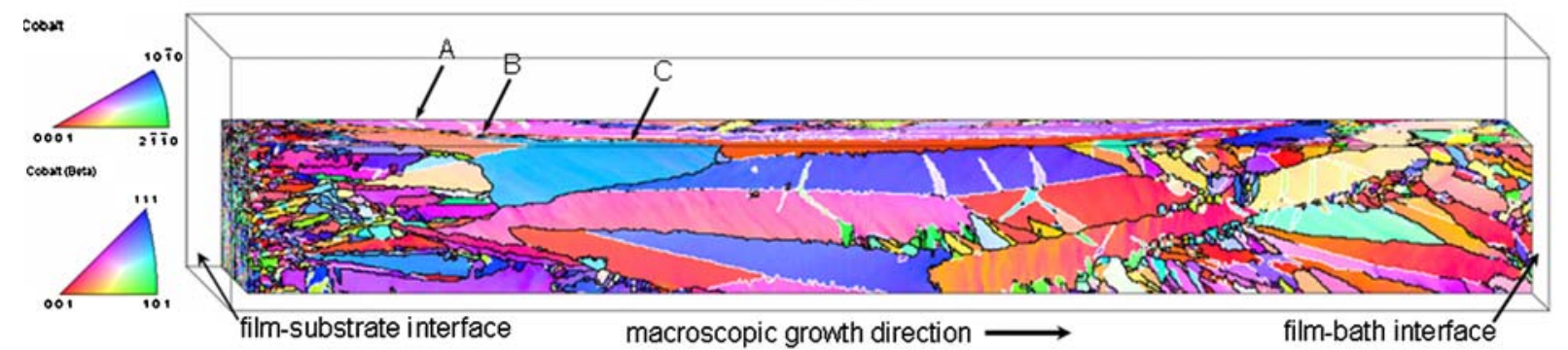

(b)

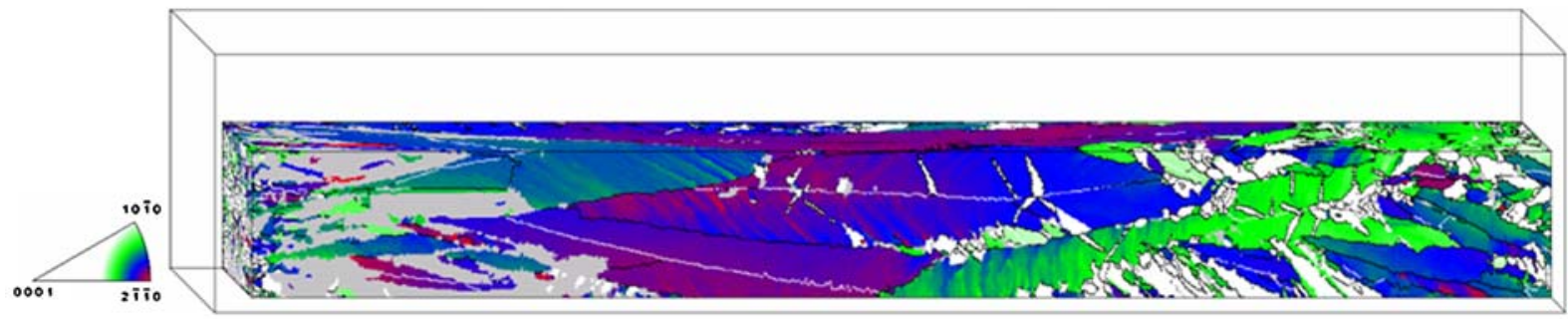

(c)

Fig. 6-3-D orientation maps from a NiCo electrodeposited film. Measurement of voxel size is $100 \times 100 \times 100 \mathrm{~nm}^{3}$. (a) Full measured orientation map colored according to the IPF of the $x$ direction. General large-angle grain boundaries $(w>15$ deg) are displayed in black; all types of twin boundaries are in white. A part is cut out in the middle, to display the position of one twin triple, marked by a circle. (b) Orientation map as in (a), cut along the plane marked in (a). The markers A, B, and C indicate particular positions in the formation of the twin triple. Note that the scattered white pixels on top of the crystal marked A occur due to the twin boundary being almost parallel to the cutting plane. (c) Orientation map colored according to the deviation of the crystal $\langle 11$ to 20$\rangle$ direction to the macroscopic growth direction Y. Areas in gray indicate crystals with fec crystal structure.

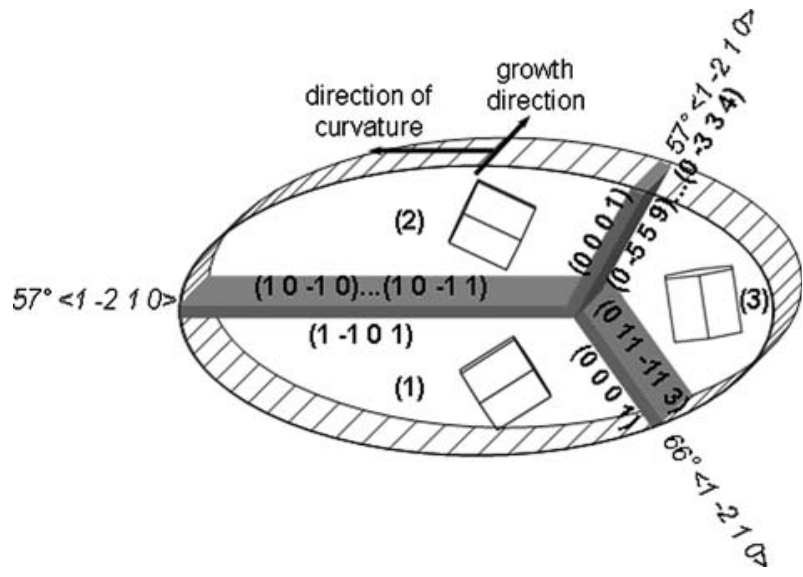

Fig. 7-Model of the twin triples frequently observed in $\mathrm{NiCo}$ deposits, as determined by 3-D orientation microscopy. The measurements allow determination of the crystal orientations, and misorientations and the crystallographic indices of all grain boundaries. Crystals (1) and (2) form a coherent 57-deg $\langle 11$ to 20$\rangle$ compressiontype twin. Crystal (3) is in a noncoherent twin relation to crystal (2), but it has no special relationship with (1). Note the occurrence of basal planes as boundary planes between (2) and (3) and (1) and (3). planes that appear in the triple grain arrangement form low-energy boundaries both in the case of the incoherent twin ((2)-(3)) as well as in case of the less specific large-angle grain boundary ((1)-(3)).

\section{Deformation and Crystallographic Orientation Patterns below Nanoindents in Cu Single Crystals}

As another example of the application of the new 3-D orientation imaging method, we conducted a 3-D study at submicrometer-scale resolution on the microstructure and crystallographic texture formed during nanoindentation in single crystals. Nanoindentation is a method increasingly used to probe the local mechanical properties of materials. However, the complex deformation mechanisms taking place during this process are only poorly understood; it is, therefore, not clear how, for example, the measured data can be related to macroscopic properties of a material.

In a set of recent articles, the group of Larson et al. ${ }^{[42-45]}$ presented the results of the microstructure 
evolution below microindents, using a nondestructive 3-D synchrotron diffraction method. In their work, the authors observed a systematic deformation-induced orientation pattern below indents in $\mathrm{Cu}$ single crystals with a (111) indentation plane normal. The experimentally observed patterns were characterized by outward rotations at the rim of the indent (tangent zone of the indent) and inward rotations directly below the indent close to the indenter axis. The results were discussed in terms of Kröner's concept of geometrically necessary dislocations (GNDs). Zaafarani et al. ${ }^{[25]}$ investigated orientation patterns below nanoindents in (111) $\mathrm{Cu}$ crystals using a manual 3-D EBSD method. These experiments revealed a pronounced deformation-induced 3-D lattice rotation field below the indent. In the (112) cross-sectional planes below the indenter tip, the observed deformationinduced rotation pattern was characterized by an outer tangent zone with large absolute values of the rotations and an inner zone closer to the indenter axis with small rotations. Additional similar studies on the microstructure evolution below small-scale indents were also published by other authors. ${ }^{[46-48]}$

In this work, we report on a more systematic study along these lines. The conical nanoindents were placed into a (111) $\mathrm{Cu}$ single crystal using different forces, namely, 4, 6, 8, and $10 \mathrm{mN}$.

Figure 8 shows a $3-\mathrm{D}$ view of the volume around the four different (111) indents. A sequence of sections for the 3-D orientation maps were taken perpendicular to the indenter axis. Figure 8(a) shows the EBSD pattern quality in the vicinity of the indents, in terms of a gray scale. The pattern quality is a general measure for the local defect density. Figure 8(b) shows the kernel average misorientation (KAM) as a measure for strongly localized orientation gradients; it also indicates the density of GNDs. It is calculated as the average of all the misorientation angles of the pixels from a "ring" surrounding a center pixel and this pixel at the center of the kernel. The KAM maps indicate the occurrence of local crystal rotations. Figure 8(c) reveals the orientation difference relative to a common reference orientation. Hence, the latter image reveals the absolute magnitude of the deformation-induced lattice rotations, which amounts to up to $15 \mathrm{deg}$. Figure 8(d) shows in more detail the lattice rotations directly under the indenter axis in a (112) plane perpendicular to the indented (111) plane: the sense of rotation changes several times, depending on the position. In accordance with the mirror symmetry parallel to the (011) face, lattice rotations are mirrored on opposite sites of the indenter. Following a tangential vector along the dashed circle in Figure 8(c), indent 3, the magnitude of the lattice rotation is highest in the $\pm[011]$ directions and slowly fades away while approaching the \pm [112] directions. The sense of rotation remains constant if one follows the tangential vector.

A particularly interesting feature is visible in Figure 8(b), which shows the existence of two concentric rings of high KAM values, indicating a high density of GNDs. The rings have the same diameter, independent of the indentation load and depth, but they appear in different depths under the surface. Since all previous investigations of the deformation and texture patterns below indents were not reproduced in full 3-D maps, as was the case in this study, these concentric reorientation rings were not yet reported in the literature. We interpret the constant diameter of these rings as being related to the direction of the radial vector on the conical surface of the indenter, creating at every surface radius a different deformation direction relative to the crystal and, therefore, activating different sets of dislocations. A more detailed analysis will be given in a subsequent article. ${ }^{[49]}$

\section{Fatigue Crack in Aluminum}

The formation of fatigue cracks is an important problem in aluminum alloys used in high-cycle fatigue conditions, e.g., in aircraft construction elements. In order to optimize alloys against fatigue, the mechanisms of damage have to be well understood; this is particularly the case with regard to microstructure and crystallography. Crack formation in the aerospace alloy Al 7075-T651 has been intensively studied by Weiland et $a l .^{[50]}$ They found that cracks start at particles close to the surface and grow into the bulk material. Since a crack is a 3-D feature, 3-D orientation microscopy is an ideal tool for studying its microstructure and crystallography. To a certain extent, the strain distribution along the crack path may also be revealed from the small orientation changes created by dislocations emitted from the crack tip during crack growth. For the observation of cracks nucleated at particles, the GIEM procedure is inadequate for the following reasons: (1) it is difficult to prepare a sample such that an appropriate crack is positioned close to a sample edge, (2) cracks developing at edges may behave very differently from those developing on the free surface, and (3) cracks at edges are frequently not observed in full. The ideal method is, therefore, LISM. Figure 9 shows the shape of the investigated sample and the measurement geometry that was used.

For the present study, 38 slices were cut and observed at a location near the center of the trench. The milling area was $34-\mu \mathrm{m}$ wide and extended over a height of more than $50 \mu \mathrm{m}$ for the last slices. Each slice had a thickness of $200 \mathrm{~nm}$, milled at $15 \mathrm{deg}$ into the surface. Orientation microscopy was carried out with a lateral step size of $200 \mathrm{~nm}$. In summary, a volume of $34 \times 50 \times 7.6 \mu \mathrm{m}^{3}$ $\left(\begin{array}{lll}x & y & z\end{array}\right)$ has been investigated with a 3-D step size of $200 \times 200 \times 200 \mathrm{~nm}^{3}$.

Figure 10(a) shows a cracked particle on the surface of the material, recorded with a backscatter detector. The particle has a surface diameter of approximately $10 \mu \mathrm{m}$. Figure 10(b) is a micrograph taken in EBSD position after eight cuts with a secondary electron detector. On the far right side, a portion of the alignment marker is visible. To the left and right sides of the observation area, small stripes are milled with a large ion current, in order to avoid shadowing. The particle is still visible in the image, but it is about to disappear. Figure 10(c) shows the area after 21 cuts: the particle has completely disappeared, but the crack, visible in Figure 10(a) as a fine white line, continues 


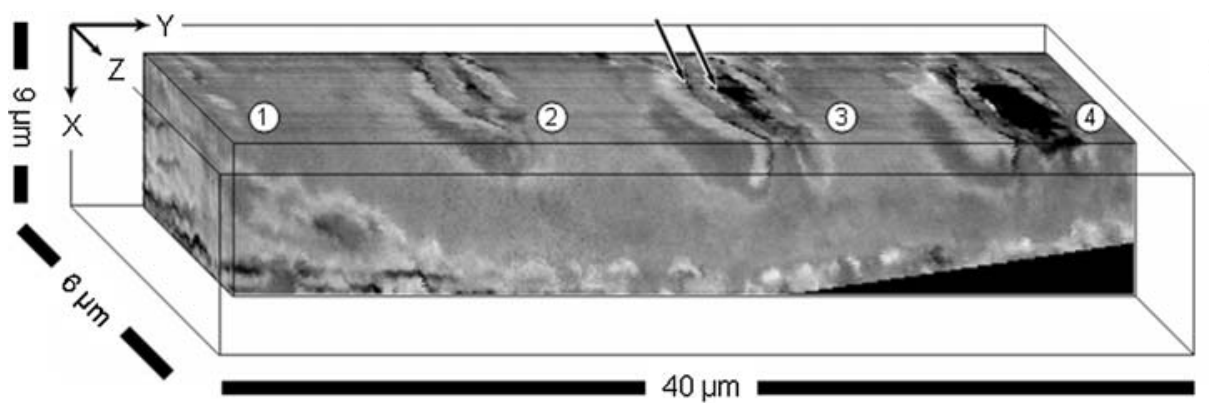

Pattern Quality

(a)

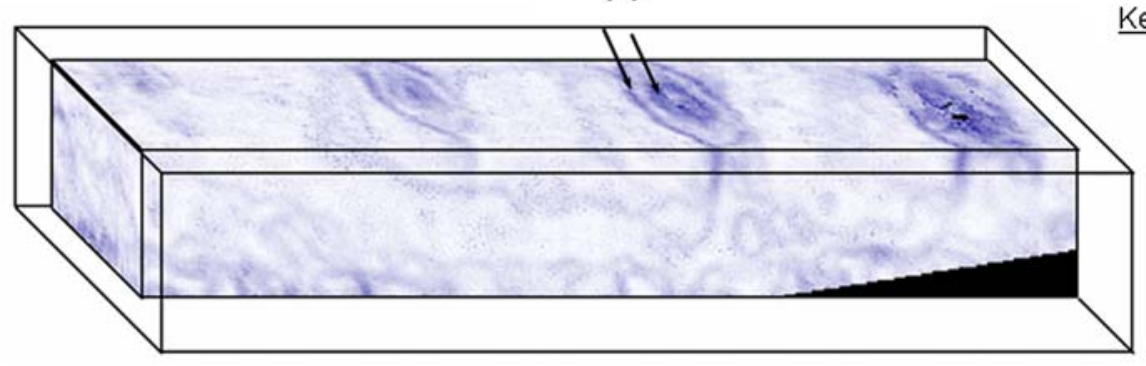

Kernel Average Misorientation

(b)

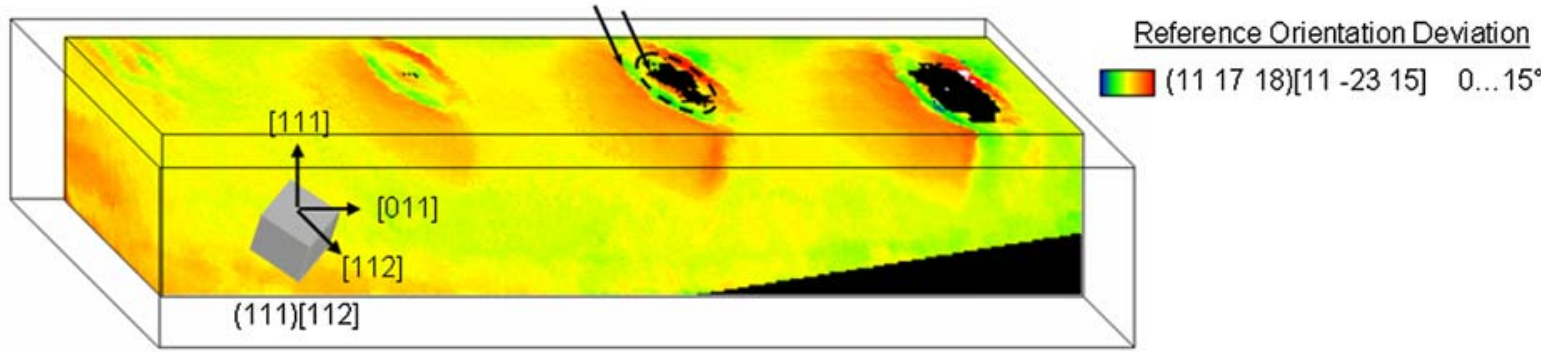

(c)
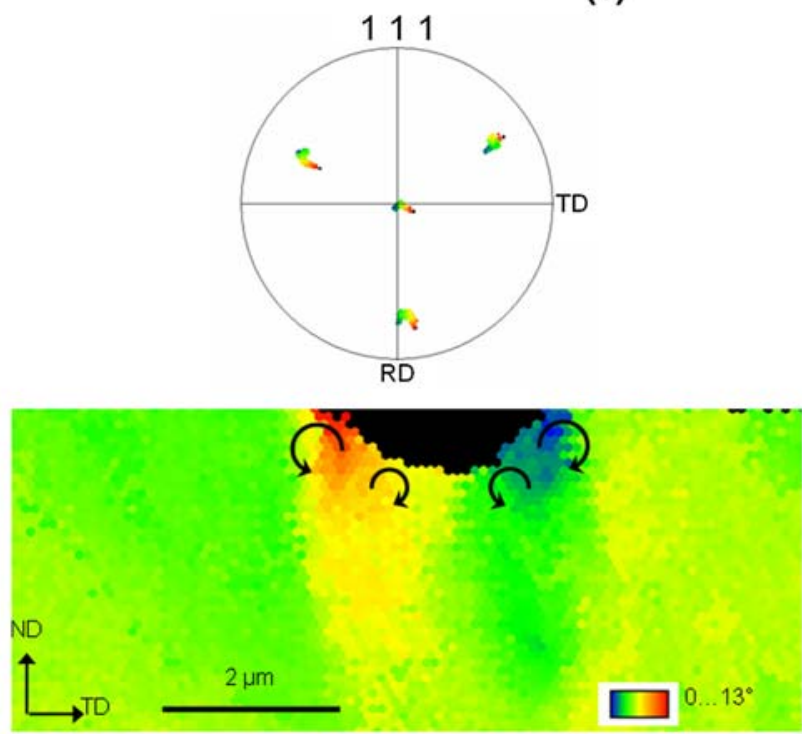

(d)

Fig. 8-3-D orientation maps of 4 nanoindents produced with a spherical indenter under loads of 4, 6, 8, and $10 \mathrm{mN}$ (indents 1 through 4). (a) Diffraction pattern quality map that serves as a rough indication of the lattice defect contents. $(b)$ KAM map, calculated up to third neighbors, with a maximum allowed misorientation of $5 \mathrm{deg}$. This map indicates local rotations due to GNDs. (c) Reference orientation deviation map. As reference, an orientation close to one side of indent 3 is selected, in order to demonstrate the asymmetry of rotations. The cube in the lower left corner indicates the orientation of the undeformed matrix (i.e., not the reference orientation). (d) Pole figures and detailed 2-D orientation map of the center part of indent 3 , as observed in $\langle 112\rangle$ direction. The colors are selected such that a unique correlation between a position in the pole figure and a position in the orientation map can be made. Arrows in the map indicate the sense of local lattice rotation. 
into the matrix material. In Figure 10(d), the appropriate orientation map (as described in the following paragraph) is shown. It indicates that the crack extends over several grains.

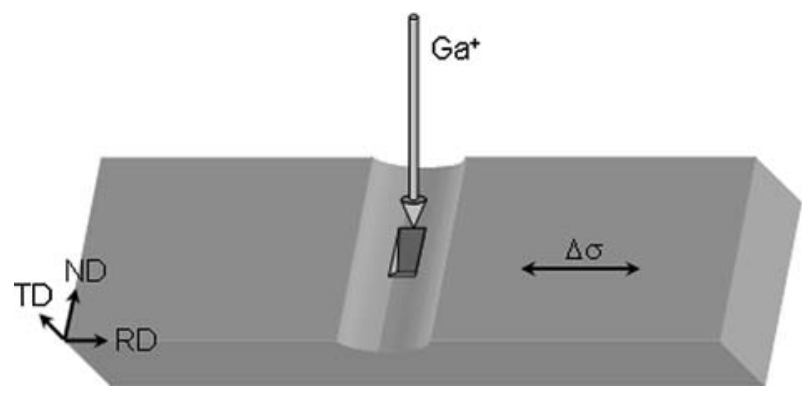

Fig. 9- Schematic of the investigated fatigue sample and position of the reference coordinate system prescribed by the cold-rolling process of the sheet. The figure is drawn from the viewpoint of the EBSD camera. The indicated ion beam mills into the surface under an angle of $15 \mathrm{deg} ; \Delta \sigma$ indicates the fatigue strain direction.
For analysis, the measured orientations were rotated such that they correspond to the reference system indicated in Figure 9. From the orientation data, microstructure maps were created: for the display of crystal orientations, IPF maps for the normal direction (ND) and rolling direction (RD) were selected. The IPF maps are overlaid with a gray value map indicating the diffraction pattern quality. Figure 11 shows the RD-IPF map of the entire characterized volume. Due to its narrowness, the crack is difficult to reveal in the orientation maps. Nevertheless, the thin area of lower image quality, highlighted by the black arrows in the 3-D map displayed in Figure 12(a), corresponds to the position of the crack. It is found that the crack continues below the particle, mainly inside of the grain that also contains the particle. It does not follow any obvious grain or subgrain boundary. The crack extends into a number of differently oriented grains, as indicated by the white arrows in the map in Figure 12(a).

In order to determine local misorientation fields, 2-D KAM maps have been calculated from the orientation data. The KAM value may be created by a regular

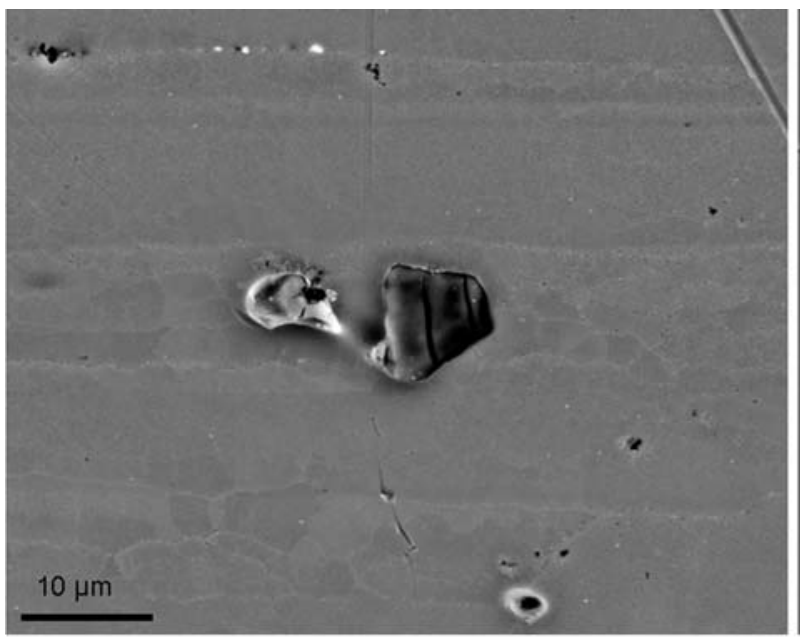

(a)

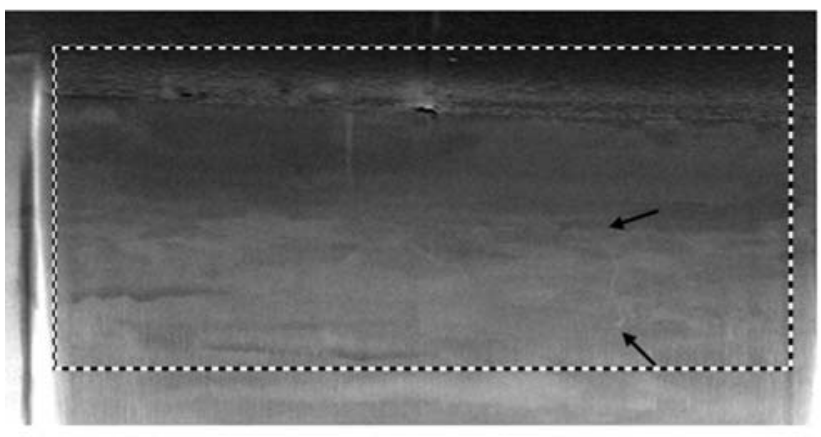

(c)

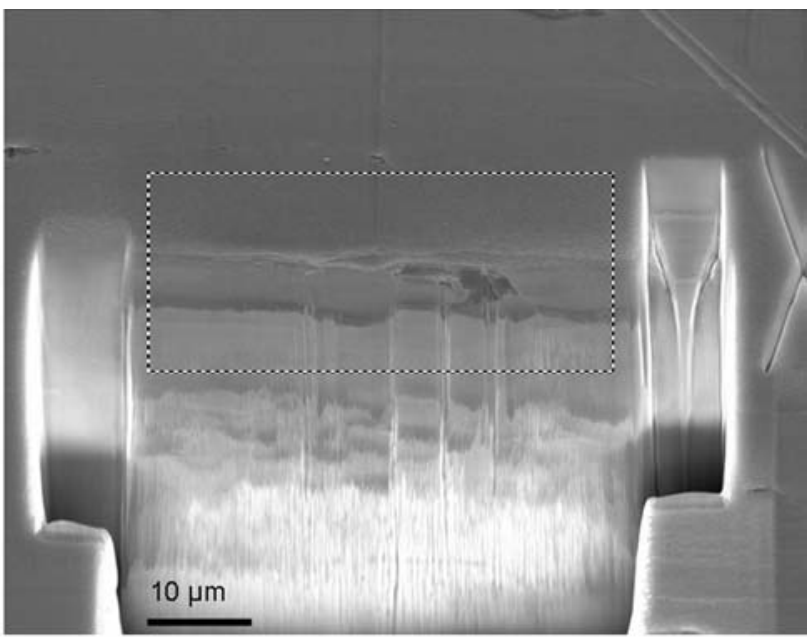

(b)

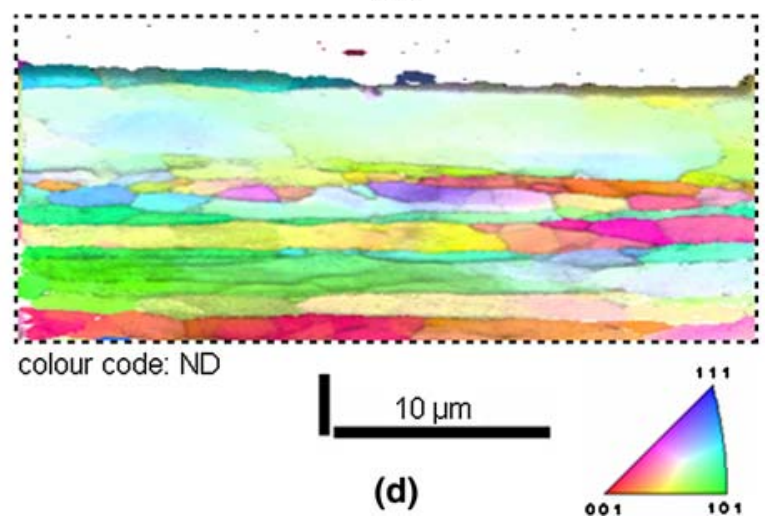

Fig. 10 - Surface view of different steps of the milling process along the crack in aluminum. (a) Backscattered electrons (BSE) micrograph of the particle on the original sample surface. (b) Milling area after eight cuts, observed with secondary electrons (SE) in EBSD position (milled area tilted $70 \mathrm{deg}$ ). Note that the original surface is under an angle of $15 \mathrm{deg}$, with respect to the milled area. The particle with crack is still visible. On the far right side, a part of the alignment marker is visible. The area for orientation microscopy is marked with a rectangle. (c) Detail of the milled area after 21 cuts. The area used for orientation microscopy is marked by a rectangle. The crack is visible as a very thin white line. The start and the end of the visible crack are marked by arrows. (d) IPF map for the ND of area (c). (Map is compressed in vertical direction to fit to the area observed under $70-$ deg tilt). 


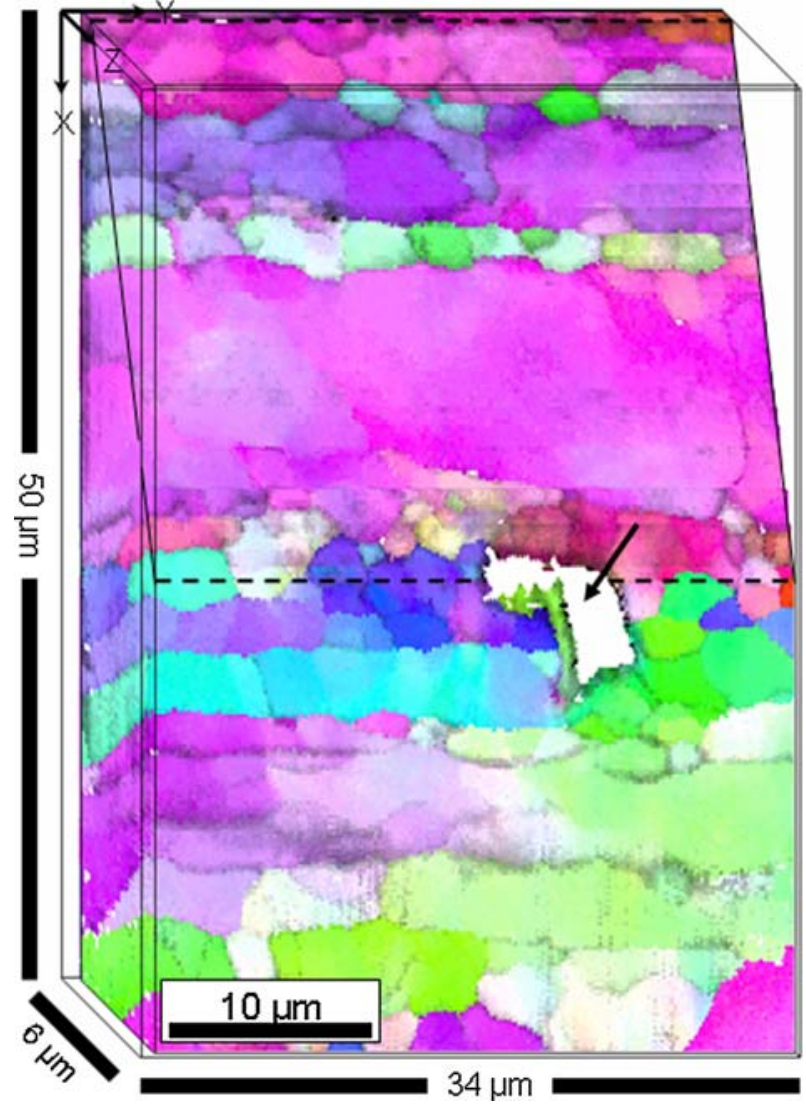

Fig. 11-3-D view of the full investigated volume. Colors according to the IPF for the rolling direction. Figure 10(d) provides the corresponding color key. The arrow indicates the position of the particle.

arrangement of dislocations - for example, a dislocation pileup at (sub)grain boundaries - and therefore may be interpreted in terms of local fields of plastic strain, as well. A 3-D view of 2-D KAM maps is shown in Figure 12(c). Areas with high KAM values are mainly small-angle grain boundaries, but the crack positions also turn out to be areas of higher local misorientations (partly marked with arrows). This may indicate that crack progression leads to the formation of regular dislocations that remain in the material.

\section{DISCUSSION: ACCURACY AND APPLICATION LIMITS}

The examples discussed here have shown that 3-D orientation microscopy based on serial sectioning with a FIB and observation of these sections by EBSD-based orientation microscopy fully preserve the powerful functions of the 2-D orientation microscopy technique and extend them into the third dimension. At each volume pixel in space, a multidimensional vector of data is obtained that includes the crystallographic phase, the crystal orientation, and an indication of the lattice defect density via EBSD pattern quality. If determined also, the elemental composition as measured by simultaneous EDS analysis may be included. In Sections A through $\mathrm{C}$, we will assess the possibilities of the technique, initially by comparison of the two possible geometrical setups described in Section II. Subsequently, we discuss the accuracy obtained by our own system; finally, we describe the material cases in which the technique cannot be applied.

\section{A. Comparison of Geometrical Setups}

Each of the two possible geometrical setups, the tilt and the rotation setup, has advantages and disadvantages. The most serious disadvantage of the tilt setup, which was developed by us, is the requirement to tilt the relatively heavy stage up or down against gravity. This leads to some inaccuracy for the tilt angle, which actually cannot be directly controlled, because it has virtually no visible effect. There are, however, easy ways to measure and correct this inaccuracy: the measurement of tilt inaccuracy is accomplished by measurement of the misorientation between neighboring points in successive slices. On samples with largely defect-free crystals, few measurement points will directly yield the inaccuracy of the tilt angle. On samples with defected crystals, the required value may be determined to be the average misorientation between all neighboring points in successive slices, excluding those that exceed a certain threshold misorientation. The correction of the tilt inaccuracy is easy, because the related distortion of the measurement field is purely linear: a wrong tilt angle leads to the expansion or compression of the measurement field in the direction perpendicular to the tilt axis. If the angle of tilt is $\alpha$ (=70 deg for EBSD) and the tilt inaccuracy is $\Delta \alpha$, the linear distortion of the measurement area is

$$
\left(l_{0}+\Delta l\right) / l_{0}=\sin \alpha / \sin (\alpha-\Delta \alpha)
$$

where $l_{0}$ denominates the true length of the measurement area, and $\Delta l$ is its distortion due to misalignment. A correction of this distortion may be easily applied to the final orientation map. As described in Section B, the tilt inaccuracy is less than $1 \mathrm{deg}$ on the present instrument. This means that the distortion is on the order of 1 pct. On a $10-\mu \mathrm{m}$-long orientation map, the extreme end of the measurement field may therefore be displaced by $100 \mathrm{~nm}$, a value which can, in most cases, be disregarded.

For the rotation setup, the most serious inaccuracy is that due to rotation. Although this inaccuracy can be measured directly by observation of two markers (it is not known to the authors whether this is actually done on these systems), the correction of this inaccuracy is less evident: a correction via beam rotation is not possible, because this leads to serious nonlinear distortions of the measurement field on the steeply inclined surface $\left(c f\right.$. the article of Nolze $\left.{ }^{[51]}\right)$. Also, a softwarebased solution is difficult, due to the nonlinear corrections required. A second disadvantage of the rotation setup is its inflexibility: different pretilted sample holders are required for the GIEM and for every LISM setup. In contrast, with the tilt setup, any tilt angle for the GIEM or LISM method can be set freely. 


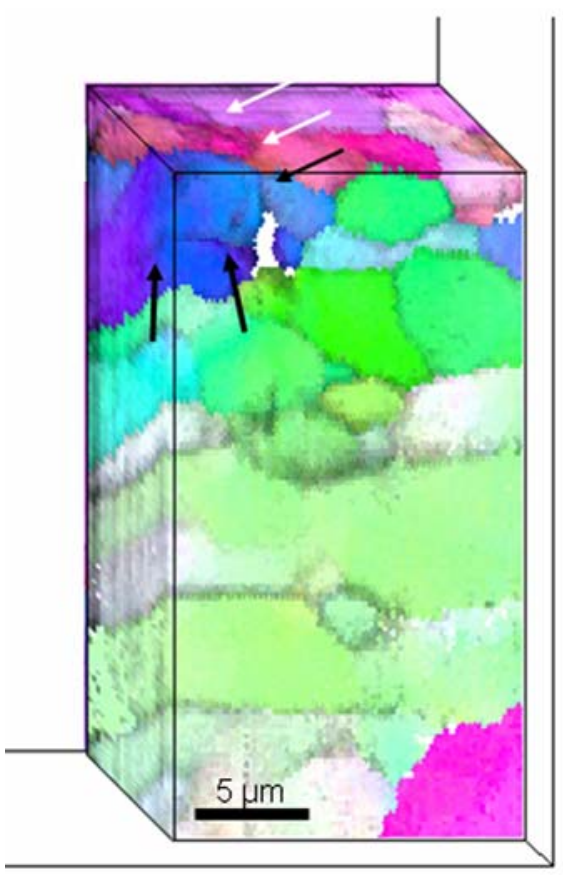

(a)

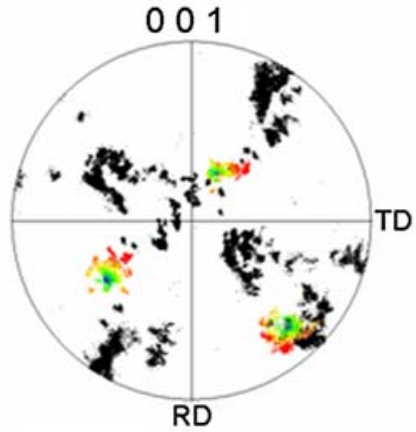

(b)

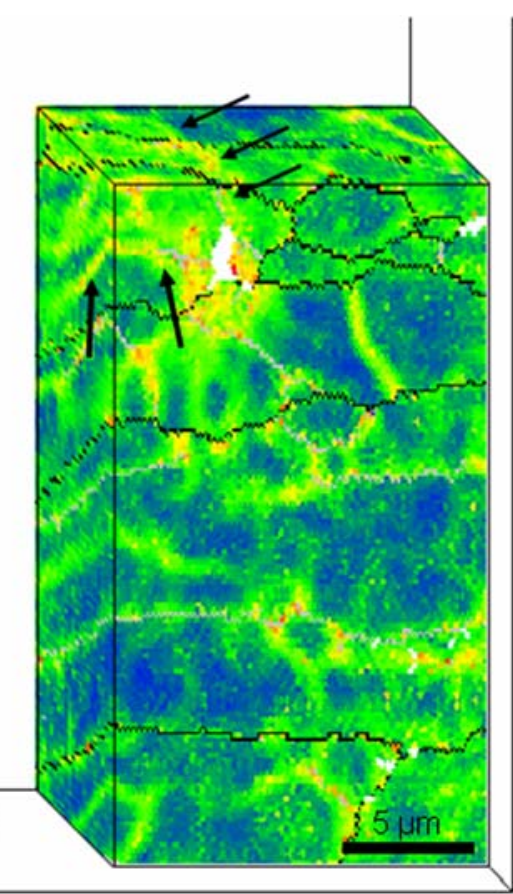

(c)

Fig. 12- (a) 3-D view of the part of the investigated volume close to the crack path. Colors according to the IPF for the rolling direction. Figure 10(d) provides the corresponding color key. (b) Pole figure of the front slice of the present view. The orientation of the main grain containing the crack path (indicated by black arrows) is indicated by color. (c) KAM map. Arrows indicate those areas of high KAM values that are due to cracks. Other areas of high KAM values are due to small-angle grain boundaries.

\section{B. Measurement Accuracy}

Important parameters for assessing the performance of the system are as follows: (1) the spatial resolution, (2) the angular resolution, and (3) the maximum size of the observable volumes with regard to the long-term stability of the instrument. We will discuss each of these parameters separately.

(1) The spatial resolution of the system results from the lateral resolution achieved on the 2-D sections, the accuracy of alignment of the sections, and the depth resolution. The lateral resolution of EBSD-based 2-D orientation microscopy has been shown to be on the order of a few $10 \mathrm{~nm}$ on a FEG SEM, depending on the material investigated and the microscope parameters used. ${ }^{[18]}$ Alignment of the serial sections is achieved by the application of image recognition software that detects the position of a marker in the image and then applies a beam shift to align this marker with a previously stored position. Image recognition and alignment is possible with an accuracy of better than 1 or 2 pixels in the digital image. On the present system, the pixel size on the viewing screen is $0.27 \mathrm{~mm}$, which corresponds to $27 \mathrm{~nm}$ at 10,000 times onscreen magnification. This value is very close to the lateral resolution of the EBSD technique. At 5000 times magnification, a resolution of 50 to $100 \mathrm{~nm}$ is achievable. A prerequisite for achieving this resolution is that the alignment marker lies within the visible image after each stage repositioning. The stage must be accurate enough to guarantee this. On the present system, the stage positioning accuracy is approximately $2 \mu \mathrm{m}$ in the $x$ and $y$ directions and $1 \mathrm{deg}$ for the tilt (point (2)), which is sufficient.

The depth resolution, i.e., the information depth of EBSD, was found to be less than $10 \mathrm{~nm} \cdot{ }^{[52]}$ This value, therefore, defines the lower limit of the achievable 3-D depth resolution, as crystallographic information from slices thinner than $10 \mathrm{~nm}$ will overlap significantly. This value is inconsequential, because the slice cutting is less precise than this. This is well known from the preparation of thin foils for TEM via FIB milling, for which it is difficult to obtain slices thinner than $50 \mathrm{~nm}$. As for the alignment of EBSD slices, the accuracy of cutting in the automated system is defined mainly by the FIB image resolution and the accuracy of the automatic image alignment via beam shift. Also, for the FIB image, the pattern recognition precision is, at best, as good as one pixel in the digital image, provided that image resolution is high enough. For a 50-pA beam, which is usually used for image alignment, image resolution is better then $20 \mathrm{~nm}$. Therefore, for a sufficiently high magnification, it is theoretically possible to reach a depth resolution of approximately $20 \mathrm{~nm}$. In reality, we found that with the currently used algorithm the image recognition is usually less precise than 1 pixel, i.e., on the order of 3 or 4 pixels. Thus, a FIB image magnification of 20,000 times is required to reliably obtain a resolution of $50 \mathrm{~nm}$. We believe that this value represents the highest resolution currently achievable. Most of the studies performed so far have been carried out with $100-\mathrm{nm}$ step sizes in all directions; this gives satisfactory section alignment. It should, however, be noted that not all image alignment 
problems have been resolved yet, as can be seen in Figure 3: the lamella arrangement of pearlite requires a particular precise alignment of the slices; otherwise, due to the narrow spacing, different lamella may be grouped together by mistake. The continuity of the lamellae is, therefore, a particularly critical measure for the precision of alignment. There are areas in which the lamellae appear perfectly aligned, particularly on the upper part of the measured block. However, other areas, at the lower part of the milled area in particular, show lamellae that cannot be uniquely grouped together. Three reasons for this misalignment have been identified. Two are related to specimen drift during the relatively slow EBSD measurements - first, due to charging of the phosphor screen of the EBSD camera and second, due to changes in environment (mainly room-temperature variations). These problems have been resolved nowadays by the exchange of the camera, which is now equipped with a thinner phosphor layer (Hikari camera, EDAX/TSL, Draper, UT) and by a better control of the air-conditioning system. The third reason is due to imperfectly flatmilled surfaces. In fact, FIB milling never leads to absolutely flat surfaces; rather, it leads to slightly curved surfaces, which also sometimes show "curtain"-like structures or small horizontal steps. Figure 13 displays a sketch of a milled surface. A slight variation in curvature, for example, due to a small variation in sample tilt, may lead to a misalignment of slices, as has been described in Section IV-A. Optimization of the milling process (optimum beam alignment and focusing, optimization of the milling time and the milled area) leads to an effective reduction in these problems.

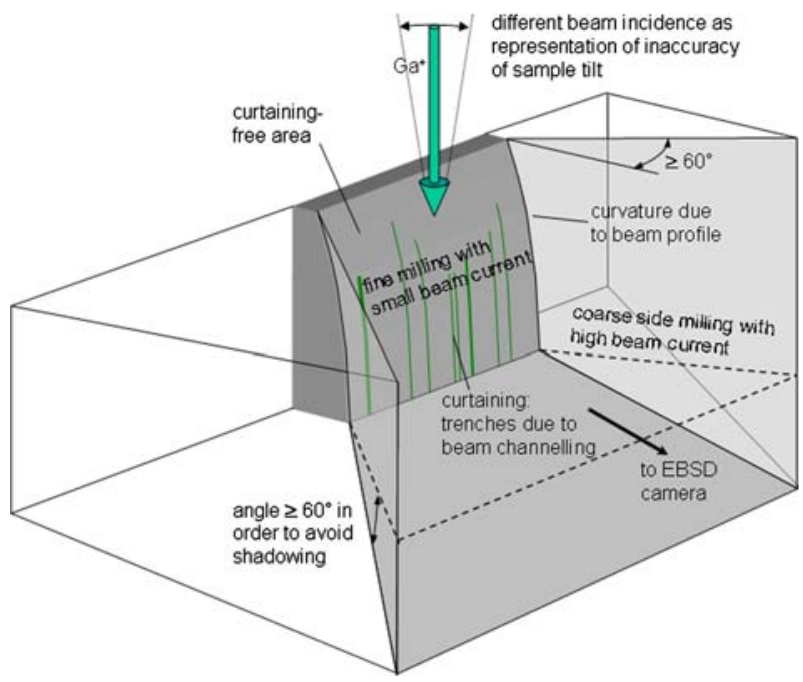

Fig. 13-Schematic drawing of the milled area indicating some geometrical features and potential sources of inaccuracy. In order to avoid shadowing the EBSD camera, the milled area has to be bounded by side walls with angles $\geq 60 \mathrm{deg}$. The large side walls may be milled with a high-beam current, while the investigated area is milled with a small-beam current. A small-beam current leads to a sharper beam profile and, therefore, to a smaller curvature of the milled surface. Inaccuracy of sample tilt (here indicated as a change of beam incidence) may lead to a significant misalignment of subsequent slices, in particular because the "natural" curvature of the surface is changed. Curtaining may also lead to local misalignment of slices.
(2) The angular resolution of the classical EBSD technique depends mainly on the size and quality of the recorded EBSD patterns. For high-speed measurements, it is on the order of 0.5 to $1 \mathrm{deg}$. The 3-D EBSD may show a larger value, due to the inaccuracy of the stage tilt. By measuring the orientation variation from slice to slice inside of a well-recrystallized grain of constant orientation, we found an average imprecision of tilt of $0.5 \mathrm{deg}$. For typical orientation microscopy applications, therefore, the angular resolution of 3-D EBSD is as good as that of the 2-D technique. Since the angular misalignment between two slices affects all pixels in a map in a similar way, it can be approximately determined - and thus corrected - by the calculation of the average misorientation of all neighboring points in neighboring slices, excluding all points that have a misorientation larger than a certain threshold value.

(3) The maximum size of the observable volume depends mainly on the material and on the time investment one wants to make. While the width of the milled surface is virtually unlimited, the height of the observable surface depends on the sensitivity of the material to curtaining. As indicated in Figure 13, from a certain distance to the top edge of the milled area, intense curtaining prevents the formation of good EBSD patterns and renders the depth position very imprecise. The distance at which curtaining starts depends strongly on the material, its microstructure and crystal orientation, the quality of the top surface, and the milling current. To date, the largest curtain-free height obtained is $30 \mu \mathrm{m}$ on a large-grained Fe3 pct Si alloy milled with a 500-pA beam current. The depth of milling, like the width, is, in principle, unlimited. However, the further one cuts into a sample, the more material has to be additionally removed, in order to prevent shadows on the EBSD screen and the serious redeposition of milled material on the sides of the milled volume. The volumelimiting parameter is, therefore, the milling time: a curtain-free area of $20 \times 20 \mu \mathrm{m}$ may be milled $1 \mu \mathrm{m}$ into a low-alloyed steel sample in approximately 15 minutes, with a beam current of $500 \mathrm{pA}$. The sides of the volume may be milled with a larger beam current at a higher rate, since they do not require any surface quality. We believe that the largest volume investigable by the method presented here is on the order of $w \times h \times d=$ $50 \times 50 \times 50 \mu \mathrm{m}^{3}$. Other groups have shown that, by using a particular sample preparation (e.g., a "finger" prepared by mechanical machining), still larger volumes can be obtained. ${ }^{[22]}$

Large volumes or high-resolution measurements require long measurement times and, therefore, the long-term stability of the instrument, in particular of the FIB gun.** With the Zeiss XB 1540, the stability of the

**Note, for example, that decreasing the step size from $100^{3}$ to $50^{3} \mathrm{~nm}^{3}$ while keeping the investigated volume constant increases the measurement time by a factor of 8 , i.e., for example, from 1 day to more than a week.

FIB gun is high enough to perform measurements lasting even as long as 10 days, providing the FIB gun is 
switched off during the EBSD measurement. After switching the FIB gun on, usually only minutes are required to obtain stable emission behavior. The stability of the gun is controlled by the automatic 3-D software, and milling is performed only after a stable emission current has been obtained.

\section{Materials Issues}

For many metals and intermetallics investigated so far (various ferritic, austenitic, and martensitic steels, nickel, copper, aluminum, FeAl, and $\mathrm{CuZnAl}$ ) excellent diffraction patterns were obtained from milled surfaces, and the microstructures observed on these were similar to those prepared by other methods. Nevertheless, various problems exist. One is the amorphization of certain materials. We have observed that $\mathrm{Nb}$-containing Laves-phase inclusions in $\mathrm{Fe}_{3} \mathrm{Al}$, which give clear diffraction patterns after mechanical preparation, do not give any patterns when prepared by milling. ${ }^{[9]}$ Similar effects have been observed on certain carbides. It is known from silicon that $\mathrm{Ga}^{+}$ion beam milling with $30 \mathrm{kV}$ ions creates an amorphous layer approximately $20 \mathrm{~nm}$ in thickness, ${ }^{[53]}$ which is too thick for EBSD. On TEM sample preparation, low- $\mathrm{kV}$ ion polishing subsequently reduces the thickness of the amorphous layer significantly. It is possible, but not yet tested, that this would also help with 3-D orientation microscopy. A second, serious problem has been observed when milling steels with metastable residual austenite-for example, low-alloyed TRIP steels. Here, all austenite transforms under the influence of the ion beam into ferrite. Up until now, this transformation could not be avoided, either by small-beam currents or by low-kV milling or a milling with $\mathrm{Ar}^{+}$ions. Finally, we have observed that the microstructure of heavily deformed aluminum may be destroyed by intense ion beam irradiation, which can probably be attributed to the formation of a low-temperature melting eutectic.

\section{CONCLUSIONS}

In recent times, a number of groups have developed systems for 3-D orientation microscopy based on automated serial sectioning via ion beam milling and EBSD-based orientation microscopy in a FIB-SEM. The technique is capable of conserving all-powerful features of the well-known 2-D EBSD-based orientation microscopy technique and extends them into the third dimension of space. It yields a multidimensional data vector for each voxel of the measured volume, including the crystal orientation, the crystallographic phase, a value for the lattice defect density, and, if measured by simultaneous EDS analysis, the elemental composition. Most systems developed so far are based on a rotation setup in which the sample has to be rotated by $180 \mathrm{deg}$ from the EBSD to the milling position. In contrast, we have developed a system based on a tilt setup that requires the sample to be tilted, in order to change between the two positions. We found that the tilt setup may be related to slightly larger sample positioning inaccuracies than the rotation setup, but the inaccuracies can be easily corrected for by software, because they create only linear image distortions. The image distortions created by the rotation setup, in contrast, are nonlinear and, therefore, are significantly more difficult to correct. Another clear advantage of the tilt method is given in terms of its larger measurement flexibility: we devised two different milling strategies that allow 3-D microstructures close to the edge of a sample or anywhere on a free surface to be investigated.

We have shown in the present article that a pixel resolution of $100 \times 100 \times 100 \mathrm{~nm}^{3}$ is obtained as a standard, but $50 \times 50 \times 50 \mathrm{~nm}^{3}$ seems to be reachable (we consider this to be a more realistic estimation than the smaller values claimed by other groups). The largest volumes observable are on the order of $50 \times 50 \times$ $50 \mu \mathrm{m}^{3}$.

The various examples presented in the work give an overview of the field of application of the technique: conductive materials with grain sizes on the order of $100 \mathrm{~nm}$ to $5 \mu \mathrm{m}$ are very accessible via the technique. In particular, heavily deformed structures can also be investigated. Furthermore, we consider the technique to be well suited for the study of all problems in which local interface properties are of importance. In contrast, we have had so far no success with the application of the technique to nonconductive materials, which is due to the fact that the charging of the sample leads to important drift problems. Furthermore, there are a number of metallic or intermetallic materials where beam-induced material changes make the application of the technique difficult or impossible. Finally, it should be mentioned that the technique is destructive and, therefore, not suited for in-situ experiments, for example. Here, the newly developed synchrotron-radiation-based 3-D techniques are of great interest. In terms of spatial resolution and observable volume, the synchrotron- and electron-based techniques appear to be largely complementary.

\section{ACKNOWLEDGMENTS}

The authors express their thanks to the people of the Zeiss hardware and software development group-in particular, to P. Hoffrogge, P. Gnauck, D. Hubbart, and H. Walaart - for their support in building up the 3-D system. The authors are also grateful to H. Weiland, Alcoa, for initiating the investigations on crack formation and delivering the appropriate sample. The authors also thank T. Takahashi, for the preparation of the pearlite sample, and N. Zaafarani, MPIE, for the preparation of the nanoindents. Finally, the authors thank J. Konrad, A. Bastos, and M. Nellessen, co-workers at MPIE, and P. Scutts, D. Dingley, and other co-workers, EDAXTSL, for their help in developing the 3-D system.

\section{REFERENCES}

1. B.L. Adams: Metall. Trans. A, 1986, vol. 17A, pp. 2199-207.

2. B.L. Adams, P.R. Morris, T.T. Wang, K.S. Willden, and S.I. Wright: Acta Metall., 1987, vol. 35, pp. 2935-46. 
3. H.F. Poulsen, S.F. Nielsen, E.M. Lauridsen, S. Schmidt, R.M. Suter, U. Lienert, L. Margulies, T. Lorentzen, and D. Juul Jensen: J. Appl. Crystallogr., 2001, vol. 34, pp. 751-56.

4. B.C. Larson, W. Yang, G.E. Ice, J.D. Budai, and J.Z. Tischler: Nature, 2002, vol. 415, pp. 887-90.

5. W. Yang, B.C. Larson, J.Z. Tischler, G.E. Ice, J.D. Budai, and W. Liu: Micron, 2004, vol. 35, pp. 431-39.

6. A. Preusser, H. Klein, and H.J. Bunge: Solid State Phenom., 2005, vol. 105, pp. 3-13.

7. E.M. Lauridsen, S. Schmidt, S.F. Nielsen, L. Margulies, H.F. Poulsen, and D. Juul-Jensen: Scripta Mater., 2006, vol. 55, pp. 51-56.

8. L. Margulies, G. Winther, and H.F. Poulsen: Science, 2001, vol. 291, pp. 2392-94.

9. J. Konrad, S. Zaefferer, and D. Raabe: Acta Mater., 2006, vol. 54, pp. 1369-80.

10. S.V. Prasad, J.R. Michael, and T.R. Christenson: Scripta Mater., 2003, vol. 48, pp. 255-60.

11. J.R. Michael, J. Schischka, and F. Altmann: HKL Technology EBSD Application Catalogue 2003, HKL Technology, Hobro, Denmark, 2003.

12. T.L. Matteson, S.W. Schwartz, E.C. Houge, B.W. Kempshall, and L.A. Gianuzzi: J. Electron. Mater., 2002, vol. 31, pp. 33-39.

13. T.T. Sheng, G.P. Goh, C.H. Tung, and L.F. Wang: J. Vac. Sci. Technol., B, 1997, vol. 15, pp. 610-13.

14. Z. Cheng, T. Sakamoto, M. Takahashi, Y. Kuramoto, M. Owari, and Y. Nihei: J. Vac. Sci. Technol., B, 1998, vol. 16, pp. 2473-78.

15. H. Jin, P.D. Wu, M.D. Ball, and D.J. Lloyd: Mater. Sci. Technol., 2005, vol. 21, pp. 419-28.

16. Electron Backscatter Diffraction in Material Science, A.J. Schwartz, M. Kumar, and B.L. Adams, eds., Kluwer Academic/Plenum Publishers, New York, NY, 2000.

17. F.J. Humphreys: J. Mater. Sci., 2001, vol. 36, pp. 3833-54.

18. D.J. Dingley: J. Microsc., 2004, vol. 213, pp. 214-24.

19. G. Spanos: Scripta Mater., 2006, vol. 55, p. 3.

20. M.D. Uchic, M. Groeber, R. Wheeler IV, F. Scheltens, and D.M. Dimiduk: Microsc. Microanal., 2004, vol. 10, pp. 1136-37.

21. J.J.L. Mulders and A.P. Day: Mater. Sci. Forum, 2005, vols. 495-497, pp. 237-43.

22. M.D. Uchic, M.A. Groeber, D.M. Dimiduk, and J.P. Simmons: Scripta Mater., 2006, vol. 55, p. 23.

23. M.A. Groeber, B.K. Haley, M.D. Uchic, D.M. Dimiduk, and S. Gosh: Mater. Charact., 2006, vol. 57, pp. 259-73.

24. S. Zaefferer, J. Konrad, and D. Raabe: Microscopy Conf. 2005, Davos, 2005, p. 63.

25. N. Zaafarani, D. Raabe, R.N. Singh, F. Roters, and S. Zaefferer: Acta Mater., 2006, vol. 54, pp. 1863-76.

26. J.R. Kremer, D.N. Mastronarde, and J.R. McIntosh: J. Struct. Biol., 1996, vol. 116, pp. 71-76.

27. D.J. Rowenhorst, A. Gupta, C.R. Feng, and G. Spanos: Scripta Mater., 2006, vol. 55, pp. 11-16.
28. F.A. Khalid and D.V. Edmonds: Acta Mater., 1993, vol. 41, pp. 3421-34.

29. M. Durrand-Charre: Microstructures of Steels and Cast Irons, Springer, New York, NY, 2004

30. G.J. Shiflet: Mater. Sci. Eng., 1986, vol. 81, pp. 61-100.

31. D.S. Zhou and G.J. Shiflet: Metall. Trans. A, 1992, vol. 23A, pp. 1259-69.

32. G. Spanos and H.I. Aaronson: Acta Metall. Mater., 1990, vol. 38, pp. 2721-32.

33. S.A. Hackney and G.J. Shiflet: Scripta Metall., 1986, vol. 20, pp. 389-94.

34. A. Vicenzo and P.L. Cavallotti: Electrochim. Acta, 2004, vol. 49, pp. 4079-89.

35. D. Golodnitsky, Y. Rosenberg, and A. Ulus: Electrochim. Acta, 2002, vol. 47, pp. 2707-14.

36. E. Gómez, J. Ramirez, and E. Vallés: J. Appl. Electrochem., 1998, vol. 28, pp. 71-79.

37. K. Daheum, D.-Y. Park, B.Y. Yoo, P.T.A. Sumodjo, and N.V. Myung: Electrochim. Acta, 2003, vol. 48, pp. 819-30.

38. A.M. El-Sherik and U. Erb: J. Mater. Sci., 1995, vol. 30, pp. $5743-49$.

39. B.Y.C. Wu, P.J. Ferreira, and C.A. Schuh: Metall. Mater. Trans. A, 2005, vol. 36A, pp. 1927-36.

40. A. Bastos, S. Zaefferer, D. Raabe, and C. Schuh: Acta Mater, 2006, vol. 54, pp. 2451-62.

41. P.G. Patridge: Metall. Rev., 1967, vol. 12, pp. 169-94.

42. W. Yang, B.C. Larson, G.M. Pharr, G.E. Ice, and J.Z. Tischler: MRS Symp. Proc., 2003, vol. 779, p. W5.34.1.

43. B.C. Larson, W. Yang, G.E. Ice, J.D. Budai, and J.Z. Tischler: Nature, 2002, vol. 415, pp. 887-90.

44. R.I. Barabash, G.E. Ice, B.C. Larson, G.M. Pharr, K.S. Chung, and W. Yang: Appl. Phys. Lett., 2001, vol. 79, pp. 749-51.

45. G.E. Ice and B.C. Larson: Adv. Eng. Mater., 2000, vol. 2, pp. 643-46.

46. Y. Liu, B. Wang, M. Yoshino, S. Roy, H. Lu, and R. Komandri: J. Mech. Phys. Solids, 2005, vol. 53, pp. 2718-41.

47. K.K. McLaughlin, N.A. Stelmashenko, S.J. Lloyd, L.J. Vandeperre, and W.J. Clegg: Mater. Res. Soc. Symp. Proc., 2005, vol. 841, pp. 3-4.

48. D. Kiener, R. Pippan, C. Motz, and H. Kreuzer: Acta Mater., 2006, vol. 54, pp. 2801-11.

49. N. Zaafarani, D. Raabe, F. Roters, and S. Zaefferer: Acta Mater, 2008, vol. 56, pp. 31-42.

50. H. Weiland, S. Cheong, J. Nardiello, S. Zaefferer, J. Papazian, and D. Raabe: submitted to J. Fatigue.

51. G. Nolze: Ultramicroscopy, 2007, vol. 107, pp. 172-83.

52. S. Zaefferer: Ultramicroscopy, 2007, vol. 107, pp. 254-66.

53. N.I. Kato, Y. Kohno, and H. Saka: J. Vac. Sci. Technol., A, 1999, vol. 17, pp. 1201-04. 